\title{
Rééducation après prothèses totales de l'épaule anatomique et inversée
}

\author{
ROBIN PHILIPPOSSIAN ${ }^{\mathrm{a}}$, Dr FRANÇOIS LUTHI ${ }^{\mathrm{a}, \mathrm{b}}$, Pr ALAIN FARRON ${ }^{\mathrm{a}}$ et Pr HES CLAUDE PICHONNAZ ${ }^{\mathrm{a}, \mathrm{c}}$
}

Rev Med Suisse 2019; 15: 1340-9

\begin{abstract}
Cet article présente la rééducation après prothèses totales d'épaule anatomique (PTEa) et inversée (PTEi), qui se découpe en 3 phases: cicatrisation et initiation du mouvement (semaines 0-6), récupération du mouvement (7-12), renforcement et retour à l'activité (13-18). Après 6-12 mois, la plupart des patients rapportent une nette diminution des douleurs et une reprise des activités légères à modérées. La rééducation de la PTEi implique spécifiquement de renforcer le muscle deltoïde et de prévenir la luxation. Le résultat fonctionnel est légèrement inférieur pour la PTEi, qui est posée lorsque des lésions musculo-tendineuses sont associées à des lésions osseuses, mais la satisfaction est comparable entre les deux types d'implants. La longévité de ces prothèses, bien que plus courte chez le patient actif jeune, est généralement bonne.
\end{abstract}

\section{Update on the rehabilitation following anatomic and reverse total shoulder arthroplasty}

This article aims to present the principles of rehabilitation following anatomical and reverse total shoulder arthroplasties. The rehabilitation consist of three phases: wound healing and movement initiation (weeks 0-6), movement recovery (7-12), strengthening and return to activity (13-18). At 6 to 12 months follow-up, most patients report a substantial decrease in pain and a return to light to moderate activity level. The rehabilitation of the reverse arthroplasty specifically requires deltoid muscle strengthening and dislocation prevention. The functional outcome is slightly inferior for reverse arthroplasty, which is indicated when musculotendinous lesions are associated to bone lesions, but satisfaction rates are comparable between the two types of implants. The durability of total shoulder arthroplasties is globally satisfying, though shorter in young active patients.

\section{INTRODUCTION}

Le développement des prothèses d'épaule a débuté dans les années 50, sous la forme d'hémi-arthroplastie (HA, seule la pièce humérale était remplacée). Ensuite les prothèses totales d'épaule dites anatomiques (PTEa, où l'on remplace égale-

\footnotetext{
a Service d’orthopédie et de traumatologie, Département de l’appareil locomoteur, CHUV et Université de Lausanne, 1011 Lausanne, ${ }^{\mathrm{b}}$ Clinique romande de réadaptation, Service de réadaptation de l'appareil locomoteur, Av. Grand-Champsec 90, 1950 Sion, ${ }^{\mathrm{C}}$ HESAV Haute Ecole de Santé Vaud, HES-SO Haute école spécialisée de Suisse occidentale, Beaumont 21, 1001 Lausanne robin.philipossian@chuv.ch | luthi.françois@chuv.ch francois.Luthi@crr-suva.ch | alain.farron@chuv.ch | claude.pichonnaz@hesav.ch claude.pichonnaz@chuv.ch
}

ment la glène) ont permis de prendre en charge l'usure de la glène, qu'elle soit primaire ou secondaire. Pour finir, la reconnaissance d'omarthroses excentrées, caractérisées par une ascension de la tête humérale en raison d'une insuffisance de la coiffe des rotateurs, source de mauvais résultats et de descellements précoces des prothèses anatomiques, a impliqué le développement de la prothèse totale d'épaule inversée (PTEi). La prothèse est dite inversée car la tête de l'humérus est remplacée par une pièce concave, alors que la glène l'est par une demi-sphère (figure 1). L'objectif est de redonner un pivot stable à l'épaule afin d'optimiser l'action du deltoïde lors de l'élévation du bras. ${ }^{1,2}$ D'abord réservées aux omarthroses associées aux ruptures de coiffe, les indications de la PTEi ont été progressivement élargies: prothèse de révision, épaule pseudo-paralytique sans omarthrose, fractures complexes, etc. Le développement des PTE anatomiques et inversées ont fait chuter les indications de l'HA. ${ }^{3,4}$ L'objectif de cet article est d'informer les cliniciens sur les principes, les étapes et les résultats de la rééducation après PTEa et PTEi.

\section{PROTHÈSE TOTALE ANATOMIQUE}

La rééducation est centrée sur les activités de la vie quotidienne (AVQ), le but étant d'atteindre la meilleure fonction possible de l'épaule. Pour des patients jeunes ou très actifs, ou lors de doutes, une communication avec le chirurgien est primordiale afin de définir des objectifs réalistes sans mettre en danger la PTEa. Les étapes de la rééducation sont présentées dans le tableau 1.

\section{Phase hospitalière}

La rééducation débute le jour suivant l'opération. Une mobilisation précoce permet une récupération plus rapide des AVQ légères et de l'indépendance du patient, bien qu'elle ne modifie pas le résultat à 1-2 ans. ${ }^{5}$ Le respect de la douleur est primordial à ce stade. La pose de la prothèse induit d'office une réinsertion du muscle sous-scapulaire, qui joue un rôle essentiel dans le maintien du centrage de la tête humérale, la récupération fonctionnelle et le risque de descellement à terme. ${ }^{6}$ Il est donc fondamental de préserver sa suture tendineuse en phase initiale. ${ }^{?}$

L'information (suites et précautions postopératoires, mise en place du gilet et exercices à domicile) est importante afin de permettre une sortie de l'hôpital en toute sécurité. ${ }^{8}$ Une fiche à l'attention des patients opérés d'une PTEa est présentée dans l'annexe 1, et une version en ligne (cf. annexe 1) est disponible pour les éléments spécifiques à la PTEi. ${ }^{9-11}$ 


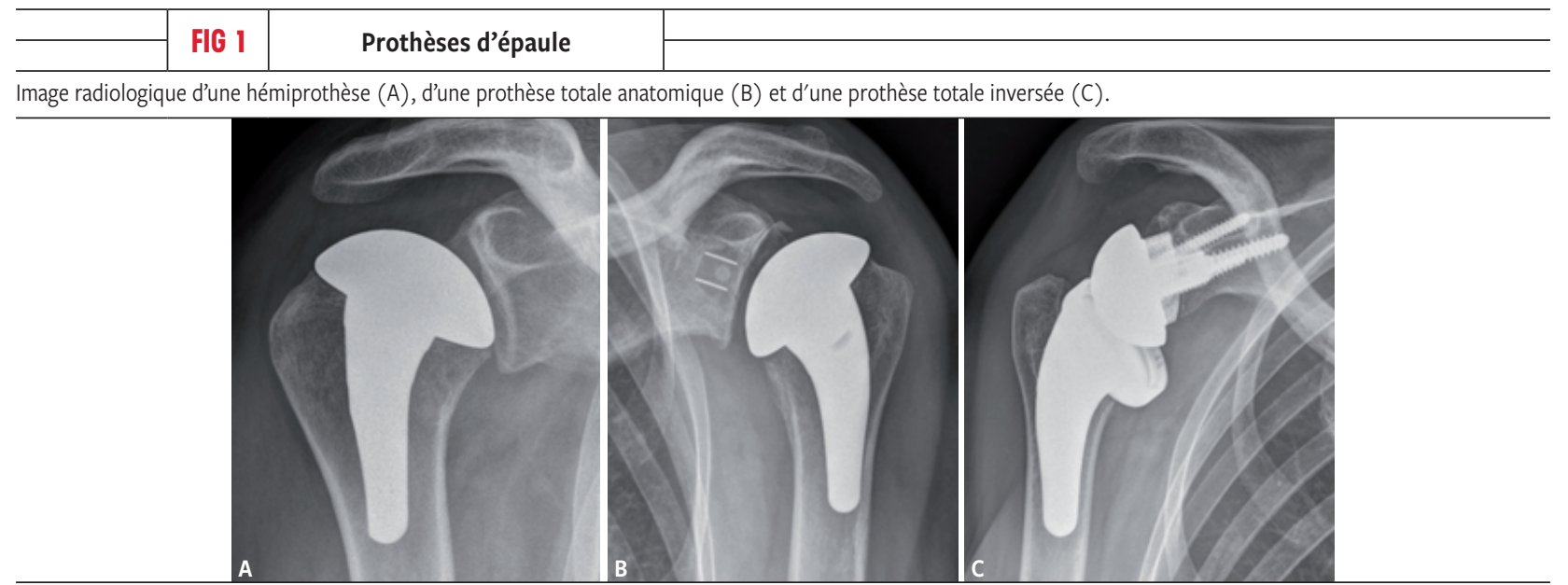

\begin{tabular}{|c|c|c|c|c|c|c|}
\hline & TABLEAU 1 & \multicolumn{3}{|c|}{ Etapes de la rééducation de la prothèse anatomique } & \multicolumn{2}{|c|}{ 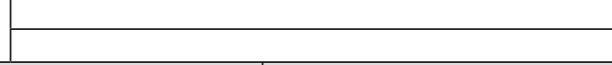 } \\
\hline Phase & \multicolumn{2}{|c|}{0 : Période hospitalière } & $\begin{array}{l}\text { 1: Cicatrisation et } \\
\text { initiation du mouvement }\end{array}$ & \multicolumn{2}{|c|}{$\begin{array}{l}\text { 2: Récupération } \\
\text { du mouvement }\end{array}$} & $\begin{array}{l}\text { 3: Renforcement } \\
\text { et retour à l'activité }\end{array}$ \\
\hline Semaines & \multicolumn{2}{|c|}{ Environ 3 jours } & $0-6$ semaines & \multicolumn{2}{|c|}{7 - 12 semaines } & 13 - 18 semaines \\
\hline $\begin{array}{l}\text { Structures anato- } \\
\text { miques concernées }\end{array}$ & \multicolumn{2}{|c|}{$\begin{array}{l}\text { - Communication opérateur } \\
\text { - Gestes chirurgicaux associés } \\
\text { - Réinsertion du sous-scapulaire } \\
\text { d’office }\end{array}$} & - Résistance faible os et tendons & \multicolumn{2}{|c|}{$\begin{array}{l}\text { - Tendon } 30 \% \text { à } 6 \text { semaines } \\
\text { • Intégration osseuse }\end{array}$} & $\begin{array}{l}\text { - Tendon } 50 \% \text { à } 12 \text { semaines } \\
\text { - Consolidation osseuse }\end{array}$ \\
\hline Type de travail & \multicolumn{2}{|c|}{$\begin{array}{l}\text { - Education du patient } \\
\text { - Mobilisation active assistée } \\
\text { et active épaule en infra-dou- } \\
\text { loureux } \\
\text { - Mobilisation en pendulaire } \\
\text { - Mobilisation active/passive } \\
\text { coude-poignet-main } \\
\text { - Evaluation sensitivomotrice }\end{array}$} & $\begin{array}{l}\text { - Mobilisation active assistée } \\
\text { et active épaule } \\
\text { - Mobilisation en pendulaire } \\
\text { - Travail actif et étirement } \\
\text { de la ceinture scapulaire } \\
\text { - Mobilisation active/passive } \\
\text { coude-poignet-main } \\
\text { - Selon besoin drainer l'œdème }\end{array}$ & \multicolumn{2}{|c|}{$\begin{array}{l}\text { - Mobilisation active assistée } \\
\text { et active épaule } \\
\text { - Tonification isométrique sous- } \\
\text { maximale infra-douloureuse } \\
\text { - Proprioception chaîne ciné- } \\
\text { tique ouverte ou chaîne fermée } \\
\text { sans charge (p.ex. ballon contre } \\
\text { un mur) }\end{array}$} & $\begin{array}{l}\text { - Mobilisation active et passive } \\
\text { de l'épaule } \\
\text { - Proprioception } \\
\text { - Renforcement concentrique } \\
\text { et excentrique du complexe } \\
\text { de l'épaule }\end{array}$ \\
\hline Objectifs & \multicolumn{2}{|c|}{$\begin{array}{l}\text { - Antalgie } \\
\text { - Assimilation des précautions } \\
\text { - Assimilation des mesures } \\
\text { d'autotraitement } \\
\text { - Initiation de la mobilisation } \\
\text { active } \\
\text { - Maintien des amplitudes } \\
\text { coude-poignet-main }\end{array}$} & $\begin{array}{l}\text { - Diminuer la douleur } \\
\text { et l'œdème } \\
\text { - Maintien des amplitudes } \\
\text { coude-poignet-main } \\
\text { - Pas d'objectif de gain d'ampli- } \\
\text { tude, viser mouvement fonc- } \\
\text { tionnel indolore dans de petites } \\
\text { amplitudes }\end{array}$ & \multicolumn{2}{|c|}{$\begin{array}{l}\text { - Indolence dans les activités } \\
\text { quotidiennes légères } \\
\text { - Mobilité active: flexion } \\
90-130^{\circ} \text {, abduction } 80-100^{\circ} \text {, } \\
\text { rotation latérale } 30-40^{\circ} \text {, rotation } \\
\text { médiale toucher la fesse avec } \\
\text { la main } \\
\text { - Mobilité fonctionnelle confor- } \\
\text { table et sans compensation }\end{array}$} & $\begin{array}{l}\text { - Activités de la vie quotidienne } \\
\text { sans douleur } \\
\text { - Mobilité active: flexion } \\
100-140^{\circ} \text {, abduction } 100-115^{\circ} \text {, } \\
\text { rotation latérale } 40-45^{\circ} \text {, rotation } \\
\text { médiale toucher la fesse avec } \\
\text { la main } \\
\text { - Force musculaire } 4 / 5 \text { selon } \\
\text { testing (légère résistance) }\end{array}$ \\
\hline Précautions & \multicolumn{2}{|c|}{$\begin{array}{l}\text { - Travail infra-douloureux } \\
\text { - Protection sous-scapulaire: } \\
\text { - Max } 90^{\circ} \text { Flex et abduction } \\
\text { durant } 6 \text { semaines } \\
\text { - Max } 0^{\circ} \text { rotation latérale, } \\
\text { pas de rotation médiale active } \\
\text { durant } 6 \text { semaines }\end{array}$} & $\begin{array}{l}\text { - Eviter douleur } \\
\text { - Gilet } 4 \text { sem. la nuit et } \\
\text { lors d'activités; peut être } \\
\text { enlevé pour activités dans les } \\
\text { amplitudes proches du corps } \\
\text { (toilette, habillage, douche, } \\
\text { alimentation...) } \\
\text { - Pas de mobilisation passive } \\
\text { - Aucun port de charge au-delà } \\
\text { de max. } 0,5 \mathrm{~kg}\end{array}$ & \multicolumn{2}{|c|}{ - Port de charge max. 1,5 kg } & $\begin{array}{l}\text { A long terme: } \\
\text { - Aucun mouvement actif contre- } \\
\text { indiqué, tant qu'il reste indolore } \\
\text { - Port de charge max. } 10 \text { - } 15 \mathrm{~kg} \\
\text { - Eviter les activités avec impacts } \\
\text { répétés sur le membre supérieur } \\
\text { - Sports adaptés: natation, nordic } \\
\text { walking, vélo, golf récréatif, tennis } \\
\text { récréatif... } \\
\text { - Envisager reclassement pro- } \\
\text { fessionnel pour les professions } \\
\text { lourdes }\end{array}$ \\
\hline $\begin{array}{l}\text { Détection } \\
\text { des complications }\end{array}$ & \multicolumn{2}{|c|}{ - Trouble sensitivomoteur } & $\begin{array}{l}\text { - Raideur/douleurs: suspecter } \\
\text { infection } \\
\text { - Douleur et déformation articu- } \\
\text { laire: luxation }\end{array}$ & \multicolumn{2}{|c|}{$\begin{array}{l}\text { - Raideur/douleurs: suspecter } \\
\text { rétraction capsulo-ligamentaire } \\
\text { - algodystrophie - infection } \\
\text { - Douleur et déformation articu- } \\
\text { laire: luxation }\end{array}$} & \\
\hline
\end{tabular}

\section{Cicatrisation et initiation du mouvement (0-6 semaines)}

Les buts poursuivis durant cette phase de 6 semaines sont l'antalgie, la récupération progressive de la mobilité de l'épaule et de la fonction dans les activités légères. Le port du gilet est prescrit dans un but antalgique, mais il est recommandé de réaliser des exercices de mobilisation du coude, du poignet et des doigts (figure 2). Des étirements des muscles qui relient l'épaule à la nuque sont utiles pour prévenir des douleurs secondaires (figure 3 ). 
La résistance des tendons suturés restant faible à ce stade, on évitera toute mise en tension du sous-scapulaire jusqu'à 6 semaines postopératoires. La flexion et l'abduction sont donc limitées à $90^{\circ}$ et la rotation latérale $(\mathrm{RL})$ à $0^{\circ}$ et on évitera de solliciter activement l'épaule en rotation médiale (RM). La mobilisation en actif-assisté (figure 4), ainsi que l'autorééducation avec un travail infradouloureux et progressif en pendulaire (figure 5), actif-assisté (figure 6), ou actif (figure 7) sont primordiales. Une utilisation progressive du membre supérieur dans les AVQ légères est conseillée, afin de favoriser l'autonomie et d'éviter le développement d'une kinésiophobie (peur du mouvement). Le port de petits objets (moins de $500 \mathrm{~g}$ ) bras au corps est possible.

\section{Récupération du mouvement (7-12 semaines)}

Les semaines 7 à 12 correspondent à la phase de récupération du mouvement et d'intégration osseuse de la prothèse. Tous les mouvements actifs, dans la limite des douleurs, sont autorisés (figures 6 et 7). La fragilité du tendon reste importante et la charge maximale admise est de $1,5 \mathrm{~kg} .{ }^{12}$

La rééducation vise la récupération des amplitudes actives tout en minimisant les compensations scapulaires, le but

\begin{tabular}{l|l|l|l}
\hline & \multirow{2}{*}{ FIG 2} & $\begin{array}{c}\text { Exercices de mobilisation active } \\
\text { du membre supérieur avec le gilet }\end{array}$ & \\
\cline { 1 - 1 } & &
\end{tabular}

(Semaines 0-6)
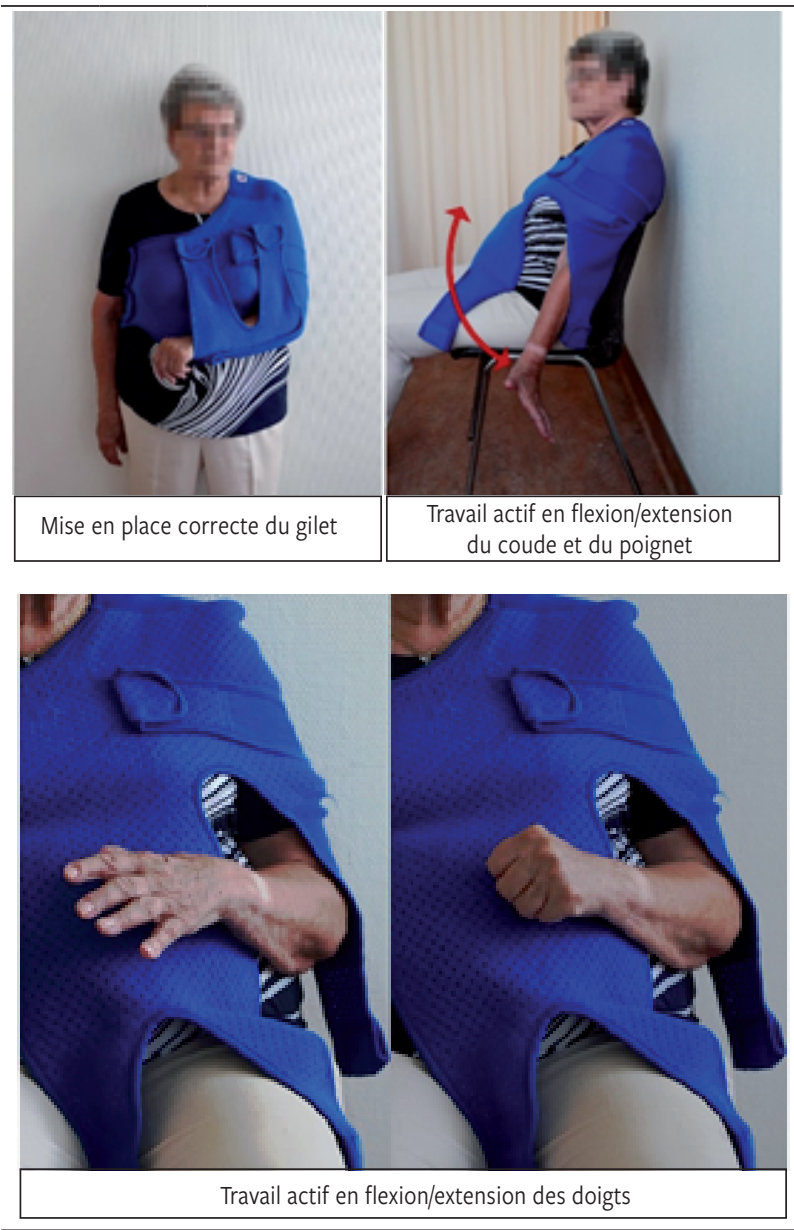

(Avec l'autorisation de la patiente).

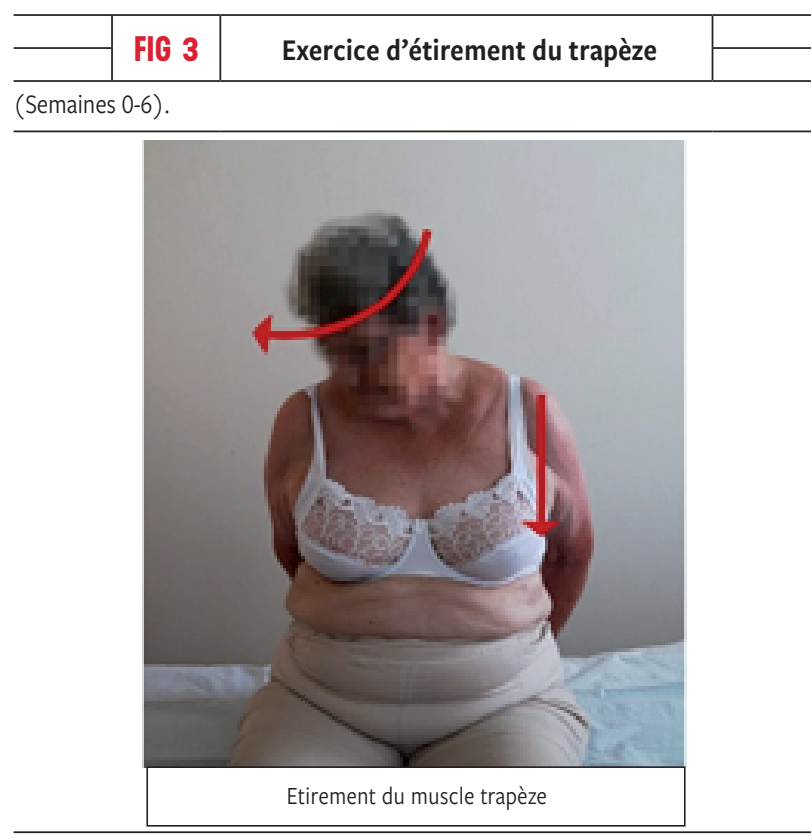

(Avec l'autorisation de la patiente).

\begin{tabular}{|c|c|c|}
\hline & \multirow{2}{*}{ FIG 4} & \multirow{2}{*}{$\begin{array}{l}\text { Mobilisation active-assistée du } \\
\text { membre supérieur de début } \\
\text { de progression }\end{array}$} \\
\hline & & \\
\hline \multicolumn{3}{|c|}{ (Semaines 0-6). } \\
\hline
\end{tabular}
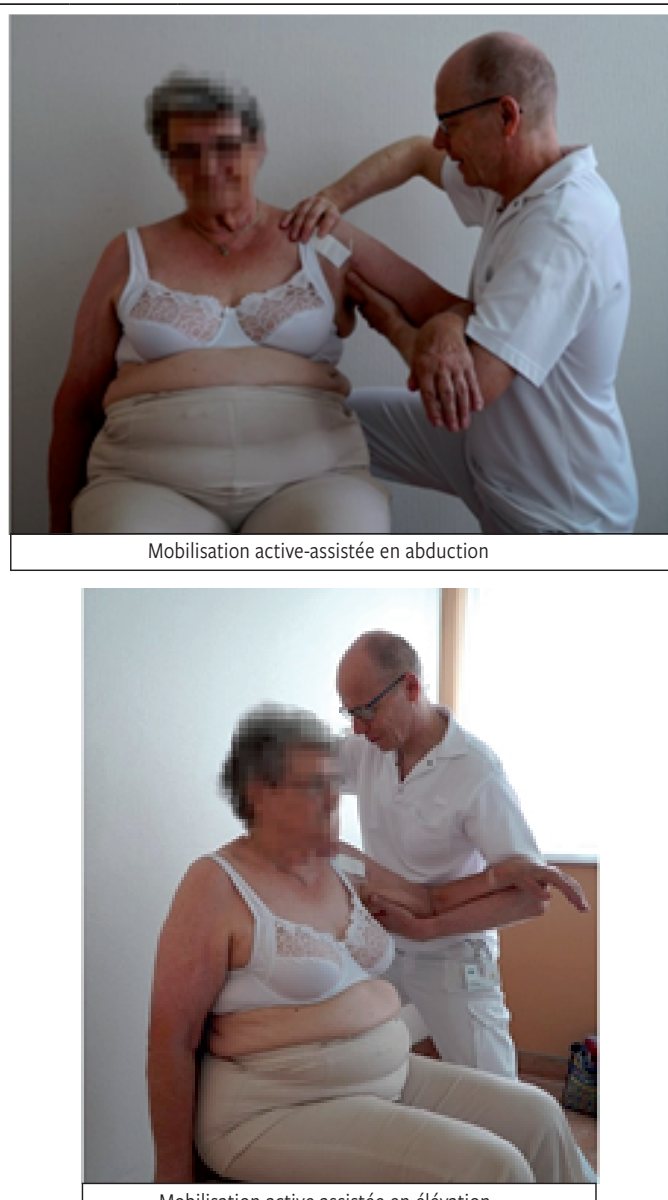

Mobilisation active assistée en élévation

(Avec l'autorisation de la patiente). 


\begin{tabular}{l|l|c|l}
\hline \multirow{2}{*}{ FIG 5} & $\begin{array}{c}\text { Exercices de mobilisation pendulaire } \\
\text { du membre supérieur de début } \\
\text { de progression }\end{array}$ & \\
\cline { 1 - 1 } & & & \\
\hline
\end{tabular}
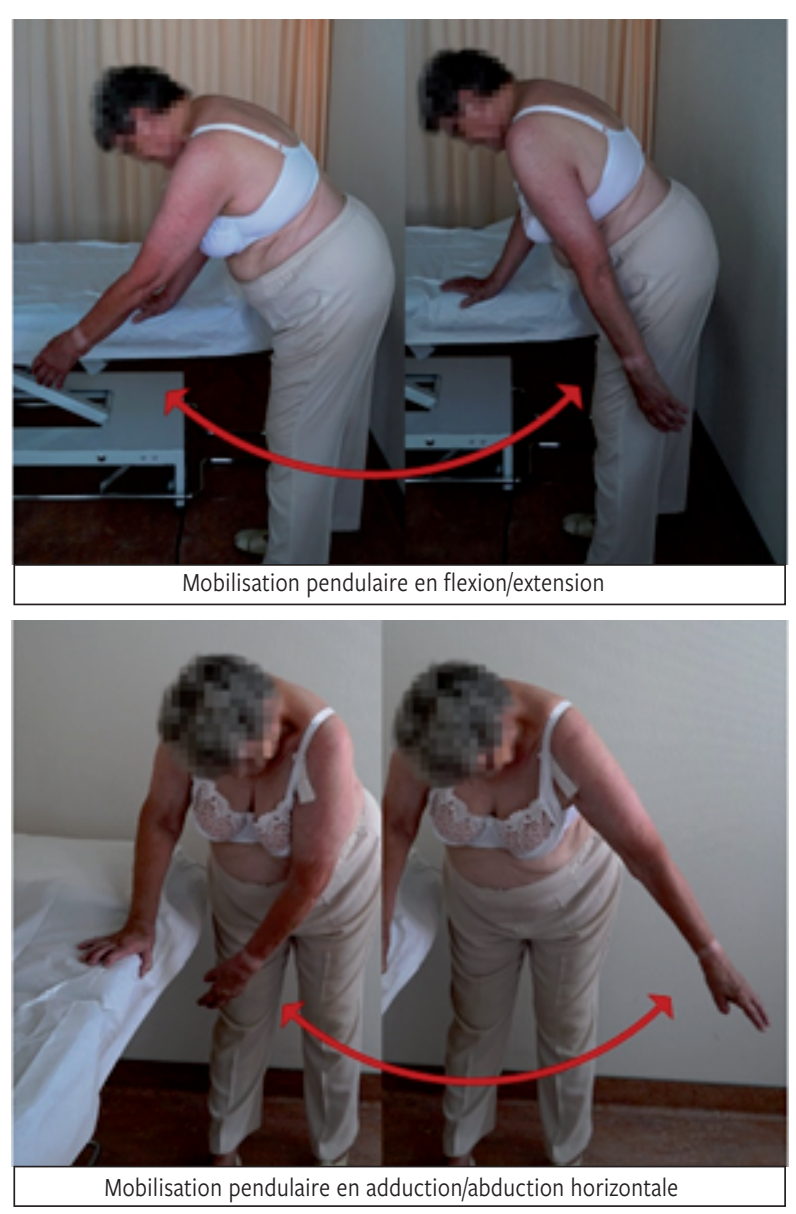

(Avec l'autorisation de la patiente).

étant de permettre au patient d'être indépendant dans les AVQ. La tonification isométrique et la proprioception peuvent débuter sans risque.

A la fin de cette phase, les activités légères ne devraient plus être douloureuses et le patient devrait être capable de réaliser les activités au-dessus de la tête.

\section{Renforcement et retour à l'activité (13-18 semaines)}

Dès la $13^{\mathrm{e}}$ semaine débute la phase de renforcement et de retour à l'activité (figure 8). Nous proposons de limiter le port de charge à 10-15 kg jusqu'à 18 semaines. Lors de la rééducation, l'accent est mis sur la proprioception et le renforcement infradouloureux. En cas de raideur qui impacte la qualité de vie du patient, une mobilisation passive à but d'étirement capsuloligamentaire peut être envisagée, en concertation avec le chirurgien.

Le patient devrait à ce stade pouvoir effectuer sans douleurs toutes ses activités quotidiennes, y compris celles au-dessus du niveau des épaules. A long terme, une mobilité jusqu'à $140^{\circ}$ en élévation peut être pronostiquée en actif (tableau 2). ${ }^{13}$

\begin{tabular}{l|l|l|l}
\hline \multirow{2}{*}{ FIG 6} & $\begin{array}{c}\text { Exercices de mobilisation } \\
\text { active-assistée du membre } \\
\text { supérieur de début de progression }\end{array}$ & \\
& & \\
\hline (Semaines 0-6).
\end{tabular}

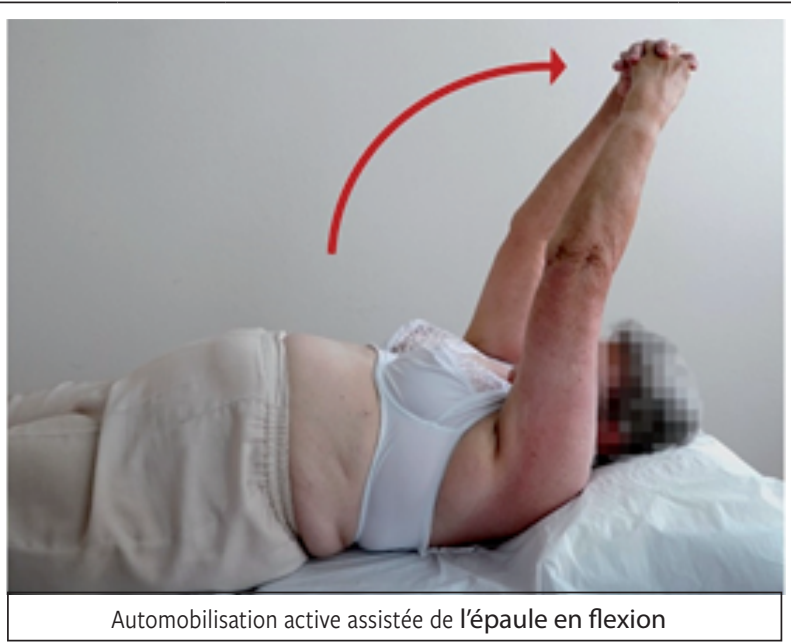

Automobilisation active assistée de l'épaule en flexion

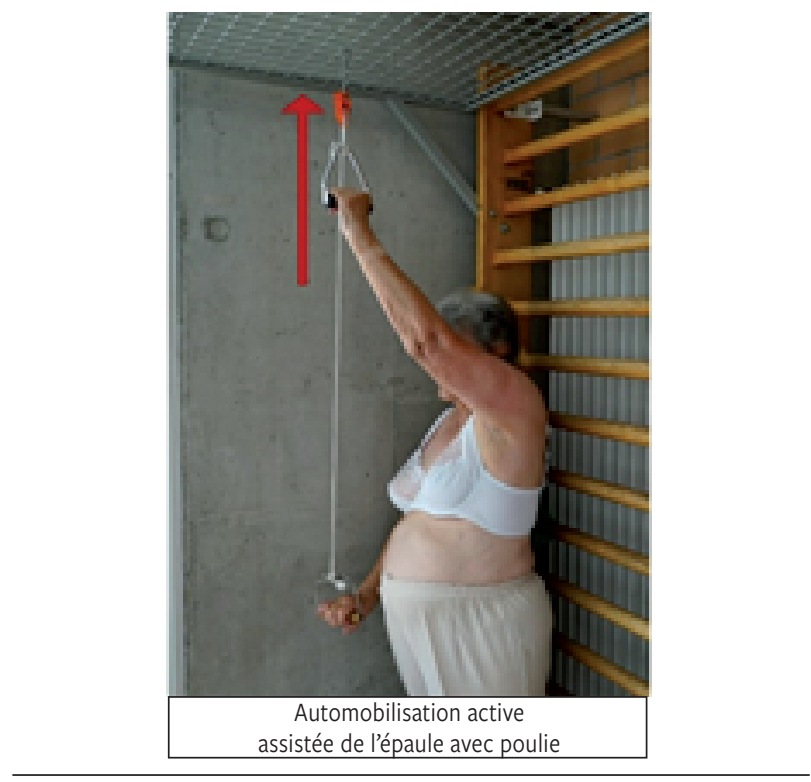

(Avec l'autorisation de la patiente).

\section{PROTHĖSE TOTALE INVERSÉE}

Les étapes de la rééducation de la prothèse inversée sont identiques à celles de la prothèse totale. Nous allons donc présenter ici les aspects spécifiques de la PTEi, en mentionnant les raisons pour lesquelles des différences existent. Les étapes de la rééducation sont présentées dans le tableau $3 .{ }^{10}$

\section{Période hospitalière}

L'abord delto-pectoral, le plus courant, implique une section du tendon du sous-scapulaire, sans réinsertion systématique, la prothèse étant par définition conçue pour une coiffe déficiente. Aucune limitation orthopédique n'est requise lors de PTEi standard. Un transfert éventuel du grand dorsal pour retrouver une rotation latérale active implique d'éviter le renforcement spécifique de ce muscle durant 4 semaines. 

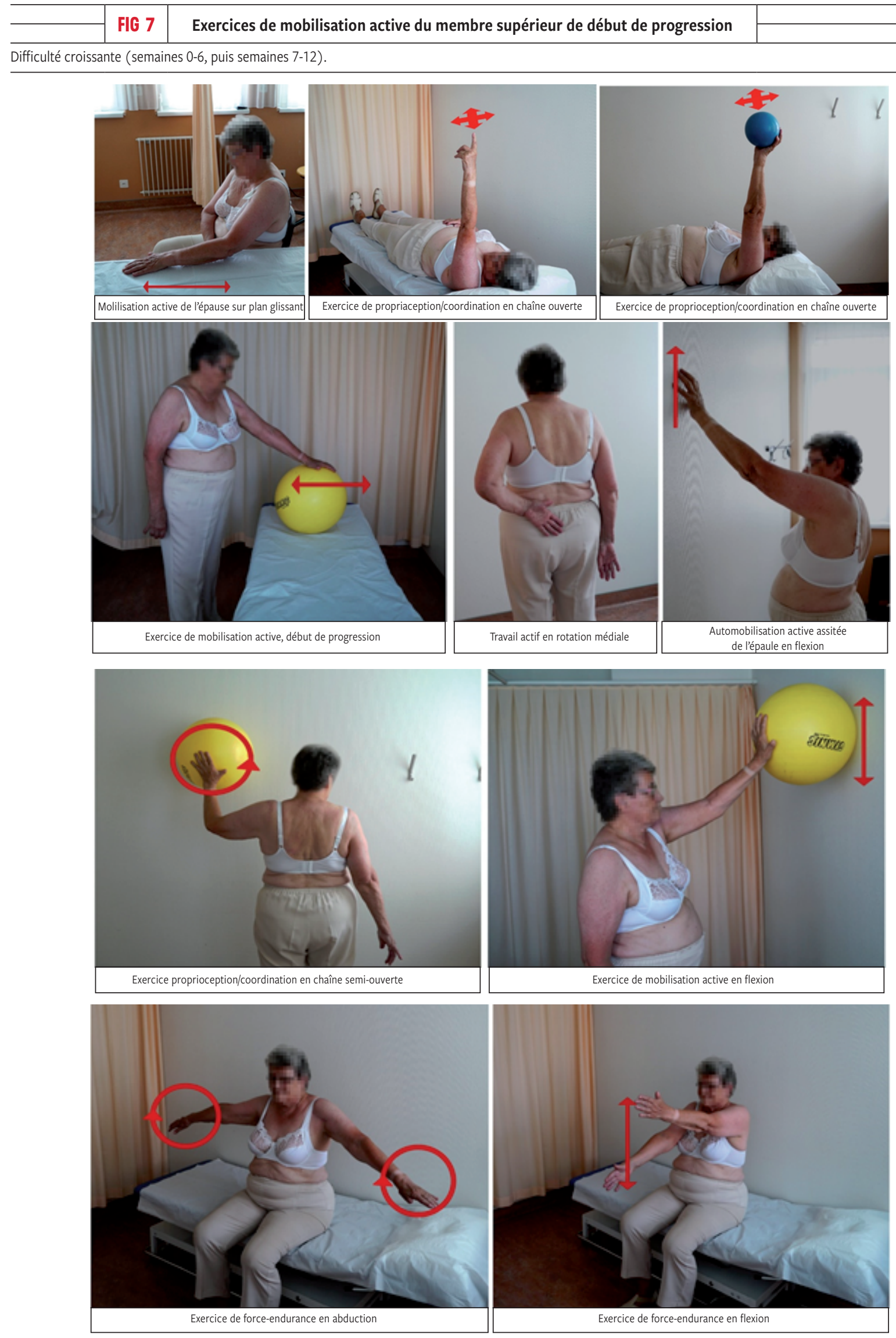

(Avec l'autorisation de la patiente). 

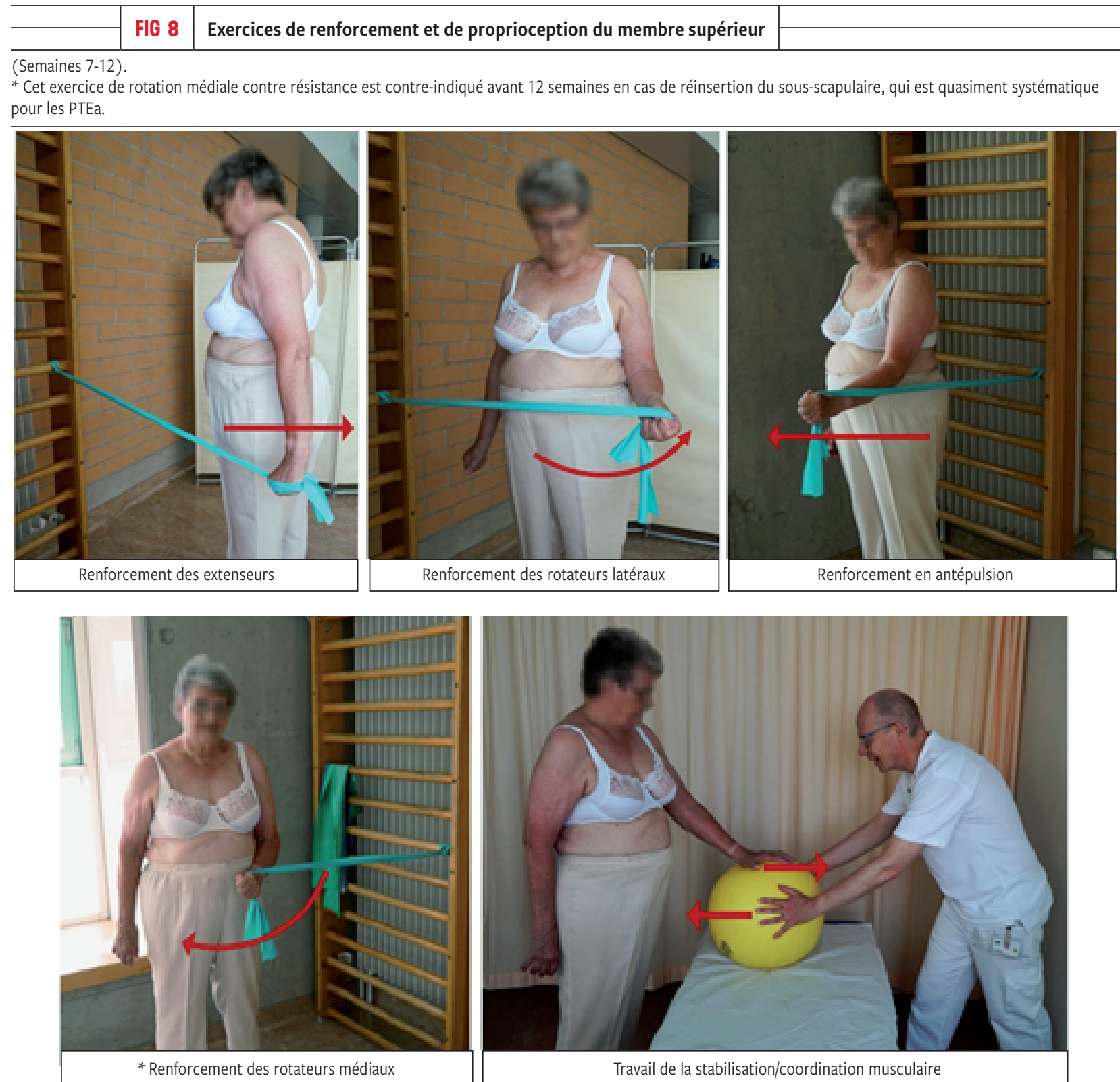

(Avec l'autorisation de la patiente).

\section{Cicatrisation et initiation du mouvement (0-6 semaines)}

Spécifiquement pour les PTEi, la mobilisation passive en extension, adduction et rotation médiale combinées est proscrite, car elle engendre un risque de luxation. Ce mouvement peut néanmoins être rééduqué sans risque majeur de manière active (figure 7 , travail actif en rotation médiale). Les autres éléments de la rééducation sont similaires à ceux de la PTEa (figures 2 à 7).

\section{Récupération du mouvement ( $7-12$ semaines)}

Le PTEi étant indiquée en cas de déficience de la coiffe des rotateurs, la tonification du deltoïde revêt une importance particulière pour pallier aux déficiences des autres muscles. Le gain d'amplitude active et l'initiation du renforcement sont centraux lors de cette phase de rééducation (figures 6 et 7). Il ne faut cependant pas perdre de vue que l'amplitude pronostiquée est généralement plus basse que celle des PTEa pour des raisons biomécaniques, bien que des compensations puissent se faire dans l'articulation scapulo-thoracique. ${ }^{14-17} \mathrm{Il}$ est donc contre-productif de rechercher un gain d'amplitude supplémentaire lorsqu'une butée se fait sentir en fin de course articulaire. La mobilité peropératoire étant un facteur prédictif de la mobilité à une année, il est utile de la connaitre pour fixer des objectifs de rééducation adéquats. ${ }^{18}$

\section{Renforcement et retour à l'activité (13-18 semaines)}

Les objectifs en termes d'amplitude articulaire étant plus modestes pour la PTEi que la PTEa, l'objectif recherché est principalement la récupération d'une fonction indolore dans les AVQ. Il est donc important d'informer le patient dès le stade préopératoire que les amplitudes se situeront aux 


\section{TABLEAU 2 Tableau comparatif des prothèses totales d'épaule anatomique ou inversée}

Généralement, les patients candidats à une prothèse inversée ont des mobilités préopératoires et des scores fonctionnels inférieurs aux patients candidats à une prothèse anatomique. En revanche, les progrès postopératoires sont souvent plus marqués pour l'élévation et la force après une prothèse inversée, alors que les gains en rotation sont plus importants après prothèse anatomique. Ce tableau indique les amplitudes que les patients peuvent atteindre en général. S’il faut 6-12 mois pour obtenir de meilleures fonction et mobilité, on peut aussi assister à une lente dégradation 5 ans après l'implantation. Score de Constant: score fonctionnel composite associant douleur, activités quotidiennes, mobilité et force.

\begin{tabular}{|c|c|c|c|c|}
\hline & $\begin{array}{l}\text { Mobilités postopératoires } \\
\text { (moyennes) }\end{array}$ & $\begin{array}{l}\text { Score de Constant } \\
(0-100 \%)^{13} \\
\text { postopératoire moyen }\end{array}$ & $\begin{array}{l}\text { Taux de survie } \\
\text { de l'implant }^{36-38}\end{array}$ & Complications \\
\hline Prothèse d'épaule anatomique & $\begin{array}{l}\text { Elévation: } 100-145^{\circ} \\
\text { Abduction: } 100-115^{\circ} \\
\text { Rotation latérale: } 40-45^{\circ}\end{array}$ & Environ $70 / 100$ & $\begin{array}{l}5 \text { ans: } 95-96 \% \\
10 \text { ans: } 90-92 \%\end{array}$ & $5-6 \%$ \\
\hline Prothèse d'épaule inversée & $\begin{array}{l}\text { Elévation: } 100-140^{\circ} \\
\text { Abduction: } 95-105^{\circ} \\
\text { Rotation latérale: } 20-30^{\circ}\end{array}$ & Environ $70 / 100$ & $\begin{array}{l}5 \text { ans: } 93-94 \% \\
10 \text { ans: } 90-91 \%\end{array}$ & $6-7 \%$ \\
\hline
\end{tabular}

\begin{tabular}{|c|c|c|c|c|}
\hline TA & \multicolumn{2}{|c|}{ Etapes de la rééducation de la prothèse inversée } & \multicolumn{2}{|c|}{ 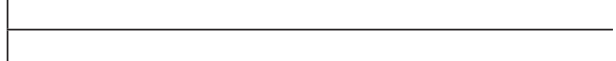 } \\
\hline Phase & $0:$ Période hospitalière & $\begin{array}{l}\text { 1: Cicatrisation et } \\
\text { initiation du mouvement }\end{array}$ & $\begin{array}{l}\text { 2: Récupération } \\
\text { du mouvement }\end{array}$ & $\begin{array}{l}\text { 3: Renforcement et retour } \\
\text { à l'activité }\end{array}$ \\
\hline Semaines & Environ 3 jours & $0-6$ semaines & 7-12 semaines & $13-18$ semaines \\
\hline $\begin{array}{l}\text { Structures anato- } \\
\text { miques concernées }\end{array}$ & $\begin{array}{l}\text { - Communication opérateur } \\
\text { - Gestes chirurgicaux associés } \\
\text { - Suture sous-scapulaire? } \\
\text { - Reconstruction osseuse? } \\
\text { - Transfert grand dorsal? } \\
\text { - Zones de sécurité }\end{array}$ & - Résistance faible os et tendons & $\begin{array}{l}\text { - Tendon } 30 \% \text { à } 6 \text { semaines } \\
\text { • Intégration osseuse }\end{array}$ & $\begin{array}{l}\text { - Tendon } 50 \% \text { à } 12 \text { semaines } \\
\text { - Consolidation osseuse }\end{array}$ \\
\hline Type de travail & $\begin{array}{l}\text { - Education du patient } \\
\text { - Mobilisation assistée et active } \\
\text { épaule en infra-douloureux } \\
\text { - Mobilisation en pendulaire } \\
\text { - Activation et étirement } \\
\text { de la musculature de la ceinture } \\
\text { scapulaire } \\
\text { - Mobilisation active/passive } \\
\text { coude-poignet-main } \\
\text { - Selon besoin : drainer l'œdème }\end{array}$ & $\begin{array}{l}\text { - Mobilisation active-assistée } \\
\text { et active de l'épaule } \\
\text { - Mobilisation en pendulaire } \\
\text { - Travail actif et étirement } \\
\text { de la ceinture scapulaire } \\
\text { - Mobilisation active/passive } \\
\text { coude-poignet-main } \\
\text { - Selon besoin : drainer l'œdème }\end{array}$ & $\begin{array}{l}\text { - Mobilisation active-assistée } \\
\text { et active de l'épaule } \\
\text { - Tonification isométrique sous- } \\
\text { maximale infra-douloureuse } \\
\text { - Tonification ciblée du deltoïde } \\
\text { - Proprioception en chaîne } \\
\text { cinétique ouverte ou fermée } \\
\text { sans charge (p.ex. ballon contre } \\
\text { un mur) }\end{array}$ & $\begin{array}{l}\text { - Mobilisation active et passive } \\
\text { de l'épaule } \\
\text { - Proprioception } \\
\text { - Renforcement concentrique } \\
\text { et excentrique du complexe de } \\
\text { l'épaule, et plus spécifiquement } \\
\text { du deltoïde }\end{array}$ \\
\hline Objectifs & $\begin{array}{l}\text { - Antalgie } \\
\text { - Assimilation des précautions } \\
\text { - Assimilation des mesures } \\
\text { d'autotraitement } \\
\text { - Initiation de la mobilisation } \\
\text { active } \\
\text { - Maintien des amplitudes } \\
\text { coude-poignet-main }\end{array}$ & $\begin{array}{l}\text { - Diminuer la douleur et } \\
\text { l'œdème } \\
\text { - Maintien des amplitudes } \\
\text { coude-poignet-main } \\
\text { - Pas d'objectif de gain d'ampli- } \\
\text { tude, viser un mouvement fonc- } \\
\text { tionnel indolore dans de petites } \\
\text { amplitudes }\end{array}$ & $\begin{array}{l}\text { - Indolence dans les activités } \\
\text { quotidiennes légères } \\
\text { - Mobilité active: flexion } \\
100-120^{\circ} \text {, abduction } 80-100 \text {, } \\
\text { rotation latérale } 15-20^{\circ} \text {, rotation } \\
\text { médiale toucher la fesse avec } \\
\text { la main } \\
\text { - Mobilité fonctionnelle confor- } \\
\text { table et sans compensation }\end{array}$ & $\begin{array}{l}\text { - Activités de la vie quotidienne } \\
\text { sans douleur } \\
\text { - Mobilité active: flexion } \\
100-140^{\circ} \text {, abduction } 95-105^{\circ} \text {, } \\
\text { rotation latérale } 20-30^{\circ} \text {, rotation } \\
\text { médiale toucher la fesse avec } \\
\text { la main } \\
\text { - Force musculaire } 4 / 5 \text { selon } \\
\text { testing (légère résistance) }\end{array}$ \\
\hline Précautions & $\begin{array}{l}\text { - Port du gilet orthopédique } \\
\text { - Pas de rotation médiale } \\
\text { active, pas de rotation latérale } \\
\text { passive durant } 6 \text { semaines, } \\
\text { sauf information contraire }\end{array}$ & $\begin{array}{l}\text { - Eviter douleur } \\
\text { - Gilet } 4 \text { sem. la nuit et lors } \\
\text { d'activités; peut être enlevé } \\
\text { pour des activités dans les } \\
\text { amplitudes proches du corps } \\
\text { (toilette, habillage, douche, } \\
\text { alimentation...) } \\
\text { - Pas de mobilisation passive } \\
\text { - Risque de luxation en ext + add } \\
\text { + rotation médiale forcée } \\
\text { - Aucun port de charge au-delà } \\
\text { de max. 0,5 kg } \\
\text { - Pas de conduite automobile }\end{array}$ & - Port de charge max. 1,5 kg & $\begin{array}{l}\text { - A long terme: } \\
\text { - Port de charge max. } 10 \text { - } 15 \mathrm{~kg} \\
\text { - Eviter les activités avec impacts } \\
\text { répétés sur le membre supérieur } \\
\text { - Sports adaptés: natation, nordic } \\
\text { walking, vélo, golf récréatif, tennis } \\
\text { récréatif... } \\
\text { - Envisager un reclassement } \\
\text { professionnel pour les professions } \\
\text { lourdes }\end{array}$ \\
\hline $\begin{array}{l}\text { Détection } \\
\text { des complications }\end{array}$ & - Troubles sensitivomoteurs & $\begin{array}{l}\text { - Raideur/douleurs: suspecter } \\
\text { une infection } \\
\text { - Douleur et déformation articu- } \\
\text { laire: luxation }\end{array}$ & $\begin{array}{l}\text { - Raideur/douleurs: suspecter } \\
\text { une rétraction capsulo-ligamen- } \\
\text { taire - une algodystrophie - } \\
\text { une infection } \\
\text { - Douleur et déformation articu- } \\
\text { laire: luxation }\end{array}$ & \\
\hline
\end{tabular}


alentours de $120^{\circ}$ d'élévation du membre supérieur, et que la rotation médiale permet au mieux d'atteindre la vertèbre $\mathrm{L} 3$ avec la main. Outre le gain d'amplitude active, le renforcement et la proprioception sont nécessaires pour la récupération de la fonction (figure 8).

\section{RÉSULTAT FONCTIONNEL ET PRONOSTIC DES PTEA ET PTEI}

L'essentiel de la récupération a lieu durant les 6 mois postopératoires, une évolution étant possible jusqu'à deux ans. ${ }^{17,19}$ Il convient cependant de bien informer le patient sur le pronostic fonctionnel, et de rester réaliste sur les bénéfices attendus. L'amélioration risque en effet d'être inférieure aux attentes des patients qui présentaient une fonction préopératoire relativement préservée. ${ }^{20}$ Les patients jeunes notamment se montrent moins satisfaits que les patients plus âgés. ${ }^{21}$ Les résultats généralement atteints pour les PTEa et PTEi sont présentés dans le tableau 1.

Concernant la PTEa, une activité physique adaptée est généralement possible, pour peu qu'elle n'implique pas le port de charges lourdes ou d'impacts répétés sur l'implant. ${ }^{22} \mathrm{Un}$ reclassement professionnel devra être envisagé en cas d'activité professionnelle contraignante. Il convient également de rester prudent quant aux sports pratiqués, certaines études ayant mis en évidence un taux de complications élevé chez de jeunes actifs qui pratiquent une activité inadaptée (charges $>10 \mathrm{~kg}$, mouvements répétitifs, mouvements fréquents audessus de la tête). ${ }^{21}$ Une majorité de chirurgiens conseille donc la pratique de sports à faible impact. ${ }^{23}$

Concernant la PTEi, bien que le résultat soit considéré comme satisfaisant chez $75-90 \%$ des patients, il ne faut pas perdre de vue que l'objectif reste la récupération de la fonction dans les AVQ. ${ }^{24,25}$ La majorité des patients reprend des activités d'intensité légère à modérée pour l'épaule après une PTEi (par exemple, jogging, natation...), ${ }^{23,26,27}$ mais la littérature reste pauvre pour étayer des recommandations. ${ }^{28}$ Une reconversion doit être envisagée chez les personnes qui exercent une activité professionnelle exigeante pour l'épaule.

\section{Risques et complications des PTEa et PTEi}

Des douleurs modérées peuvent encore être présentes au stade initial. L'apparition d'une raideur ou d'une douleur suspecte peut être le signe de complications précoces. Une déformation orientera vers une possible luxation, tandis que d'autres signes cliniques (écoulement, rougeur, chaleur, fièvre) dirigeront plutôt vers une infection. En plus des complications précitées, on restera attentif à l'apparition d'une raideur secondaire de l'épaule en cas de limitation d'amplitude douloureuse. Une péjoration tardive des douleurs ou de la fonction de l'épaule implique des examens pour en détecter
3 Gallinet D, Ohl X, Decroocq L, et al. Is reverse total shoulder arthroplasty more effective than hemiarthroplasty for treating displaced proximal humerus fractures in older adults? A systematic review and meta-analysis. Orthop Traumatol Surg Res 2018;104:759-66. la cause (usure ou descellement d'un composant, érosion de la glène, dégradation de la coiffe, fracture, infection....).,29-31

La PTEi présente un taux de complications légèrement plus élevé que les autres types de prothèses: luxations, infections, neuropathies, ce qui implique de choisir de manière rigoureuse l'implant le plus adapté à la situation du patient., ${ }^{1,31}$ Les complications spécifiques de la PTEi sont liées au risque de luxation, qui reste cependant limité à $2,7 \% .^{32}$

Malgré un taux de survie $>90 \%$ à 10 ans, certaines études pointent des risques d'échec et de descellement des PTEa chez les patients de moins de 50 ans ayant un niveau d'activité élevé. ${ }^{33,34}$ La longévité de la PTEi reste également problématique chez les patients jeunes et actifs. ${ }^{35}$ En cas de révision sans possibilité de reconstruction, l'intervention consiste à convertir la PTEi en hémiarthroplastie en gardant uniquement la tige humérale.

\section{CONCLUSION}

Les prothèses d'épaule offrent des solutions thérapeutiques tout à fait satisfaisantes pour récupérer un mouvement fonctionnel et indolore dans les activités de la vie courante. Les PTEi ont des résultats légèrement inférieurs à ceux des PTEa, mais représentent une option à privilégier dans les situations où l'intégrité de la coiffe des rotateurs n'est pas assurée. La rééducation veille à être progressive, en recherchant le bon équilibre entre le respect des douleurs et la reprise des activités. La longévité de la prothèse peut s'avérer problématique chez le patient jeune. Il convient donc d'envisager une reconversion professionnelle et sportive pour préserver la prothèse chez les personnes actives.

Conflit d'intérêts: Les auteurs n'ont déclaré aucun conflit d'intérêts en relation avec cet article.

\section{IMPLICATIONS PRATIQUES}

- Les prothèses totales d'épaule constituent une option efficace pour diminuer les douleurs et améliorer la fonction en cas d'omarthrose

- La pose d'une prothèse totale inversée est indiquée en cas d'incompétence de la coiffe des rotateurs

- La rééducation se découpe en phases de cicatrisation et initiation du mouvement (0-6 sem.), récupération du mouvement (7-12 sem.) et renforcement et retour à l'activité (13-18 sem.)

- Le pose d'une prothèse inversée implique de renforcer spécifiquement le deltoïde et d'éviter les mouvements passifs en rotation médiale + extension (risque de luxation)

- Une reconversion est à discuter en cas d'activité professionnelle contraignante pour l'épaule versus delayed passive range of motion following total shoulder arthroplasty. Shoulder Elbow Surg 2016;25:1918-24. 6 Terrier A, Larrea X, Malfroy Camine V, et al. Importance of the subscapularis muscle after total shoulder arthroplasty. Clin Biomech (Bristol, Avon) 2013;28:146-50. 
7 Wright T, Easley T, Bennett J, et al. Shoulder arthroplasty and its effect on strain in the subscapularis muscle. Clin Biomech (Bristol, Avon) 2015;30:373-6 8 ** Padey $A$. La rééducation après arthroplastie de l'épaule - informations à donner aux patients. Revue du Rhumatisme Monographies 2010;77:253-63. 9 ** Payne C, Jaggi A, Le Leu A, et al. (v) Rehabilitation for shoulder arthroplasty. Orthop Trauma 2015;29:313-23.

$10 * *$ Boudreau S, Boudreau ED, Higgins $L D$, et al. Rehabilitation following reverse total shoulder arthroplasty. J Orthop Sports Phys Ther 2007;37:734-43. 11 Wilcox RB, Arslanian LE, Millett P. Rehabilitation following total shoulder arthroplasty. J Orthop Sports Phys Ther 2005;35:821-36.

12 Pichonnaz C, Milliet J, Farron A, et al. Mise au point sur la rééducation après chirurgie de la coiffe des rotateurs de l'épaule. Rev Med Suisse 2016;12:1278-83. 13 Flurin PH, Roche CP, Wright TW, et al. A Comparison and correlation of clinical outcome metrics in anatomic and reverse total shoulder arthroplasty. Bull Hosp Jt Dis (2013) 2015;73(Suppl. 1):S118-23.

14 Terrier A, Scheuber P, Pioletti DP, et al. Activities of daily living with reverse prostheses: importance of scapular compensation for functional mobility of the shoulder. J Shoulder Elbow Surg 2013;22:948-53.

15 de Toledo JM, Loss JF, Janssen TW, et al. Kinematic evaluation of patients with total and reverse shoulder arthroplasty during rehabilitation exercises with different loads. Clin Biomech (Bristol,
Avon) 2012;27:793-800.

16 Walker D, Matsuki K, Struk AM, et al. Scapulohumeral rhythm in shoulders with reverse shoulder arthroplasty. J Shoulder Elbow Surg 2015;24:1129-34.

17 Levy JC, Everding NG, Gil CC, Jr., et al. Speed of recovery after shoulder arthroplasty: a comparison of reverse and anatomic total shoulder arthroplasty. J Shoulder Elbow Surg 2014;23:1872-81. 18 Schwartz DG, Cottrell BJ, Teusink MJ, et al. Factors that predict postoperative motion in patients treated with reverse shoulder arthroplasty. J Shoulder Elbow Surg 2014;23:1289-95.

19 * Razmjou H, Stratford P, Kennedy D, et al. Pattern of recovery following total shoulder arthroplasty and humeral head replacement. BMC Musculoskelet Disord 2014;15:306.

20 Werner BC, Wong AC, Mahony GT, et al. Causes of poor postoperative improvement after reverse total shoulder arthroplasty. J Shoulder Elbow Surg 2016;25:e217-22.

21 Walters JD, Barkoh K, Smith RA, et al. Younger patients report similar activity levels to older patients after reverse total shoulder arthroplasty. J Shoulder Elbow Surg 2016;25:1418-24.

22 Kusnezov N, Dunn JC, Parada SA, et al. Clinical outcomes of anatomical total shoulder arthroplasty in a young, active population. Am J Orthop (Belle Mead NJ) 2016;45:E273-82.

23 Magnussen RA, Mallon WJ, Willems WJ, et al. Long-term activity restrictions after shoulder arthroplasty: an international survey of experienced shoulder surgeons. J Shoulder Elbow Surg 2011:20:281-9.

24 Sershon RA, Van Thiel GS, Lin EC, et al. Clinical outcomes of reverse total shoulder arthroplasty in patients aged younger than 60 years. J Shoulder Elbow Surg 2014;23:395-400.

25 Samitier G, Alentorn-Geli E, Torrens C, et al. Reverse shoulder arthroplasty. Part 1: Systematic review of clinical and functional outcomes. Int J Shoulder Surg. 2015;9:24-31.

26 Johnson CC, Johnson DJ, Liu JN, et al. Return to sports after shoulder arthroplasty. World J Orthop 2016;7:519-26. 27 Fink Barnes LA, Grantham WJ, Meadows MC, et al. Sports activity after reverse total shoulder arthroplasty with minimum 2-year follow-up. Am J Orthop (Belle Mead NJ) 2015;44:68-72. 28 Golant A, Christoforou D, Zuckerman $J D$, et al. Return to sports after shoulder arthroplasty: a survey of surgeons' preferences. J Shoulder Elbow Surg 2012;21:554-60.

29 Jacxsens M, Walz T, Durchholz H, et al. Towards standardised definitions of shoulder arthroplasty complications: a systematic review of terms and definitions. Arch Orthop Trauma Surg 2017;137:347-55.

30 Wiater BP, Moravek JE, Jr., Wiater JM. The evaluation of the failed shoulder arthroplasty. J Shoulder Elbow Surg 2014;23:745-58.

31 Alentorn-Geli E, Samitier G, Torrens C, et al. Reverse shoulder arthroplasty. Part 2: Systematic review of reoperations, revisions, problems, and complications.
Int J Shoulder Surg 2015;9:60-7.

32 Kohan EM, Chalmers PN, Salazar D, et al. Dislocation following reverse tota shoulder arthroplasty. J Shoulder Elbow Surg 2017;26:1238-45.

33 Denard PJ, Raiss P, Sowa B, et al. Mid- to long-term follow-up of total shoulder arthroplasty using a keeled glenoid in young adults with primary glenohumeral arthritis. J Shoulder Elbow Surg 2013;22:894-900.

34 Bartelt R, Sperling JW, Schleck CD, et al. Shoulder arthroplasty in patients aged fifty-five years or younger with osteoarthritis. J Shoulder Elbow Surg 2011;20:123-30.

35 * Ek ET, Neukom L, Catanzaro S, et al. Reverse total shoulder arthroplasty for massive irreparable rotator cuff tears in patients younger than 65 years old: results after five to fifteen years. $J$ Shoulder Elbow Surg 2013;22:1199-208. 36 Barlow JD, Yuan BJ, Schleck CD, et al. Shoulder arthroplasty for rheumatoid arthritis: 303 consecutive cases with

minimum 5-year follow-up. J Shoulder Elbow Surg 2014;23:791-9.

37 Fevang B-TS, Lygre SHL, Bertelsen G, et al. Good function after shoulder arthroplasty. Acta Orthop 2012;83:467-73. 38 Cuff DJ, Pupello DR, Santoni BG, et al. Reverse shoulder arthroplasty for the treatment of rotator cuff deficiency: a concise follow-up, at a minimum of 10 years, of previous reports. J Bone Joint Surg Am 2017;99:1895-9.

* à lire

** à lire absolument 


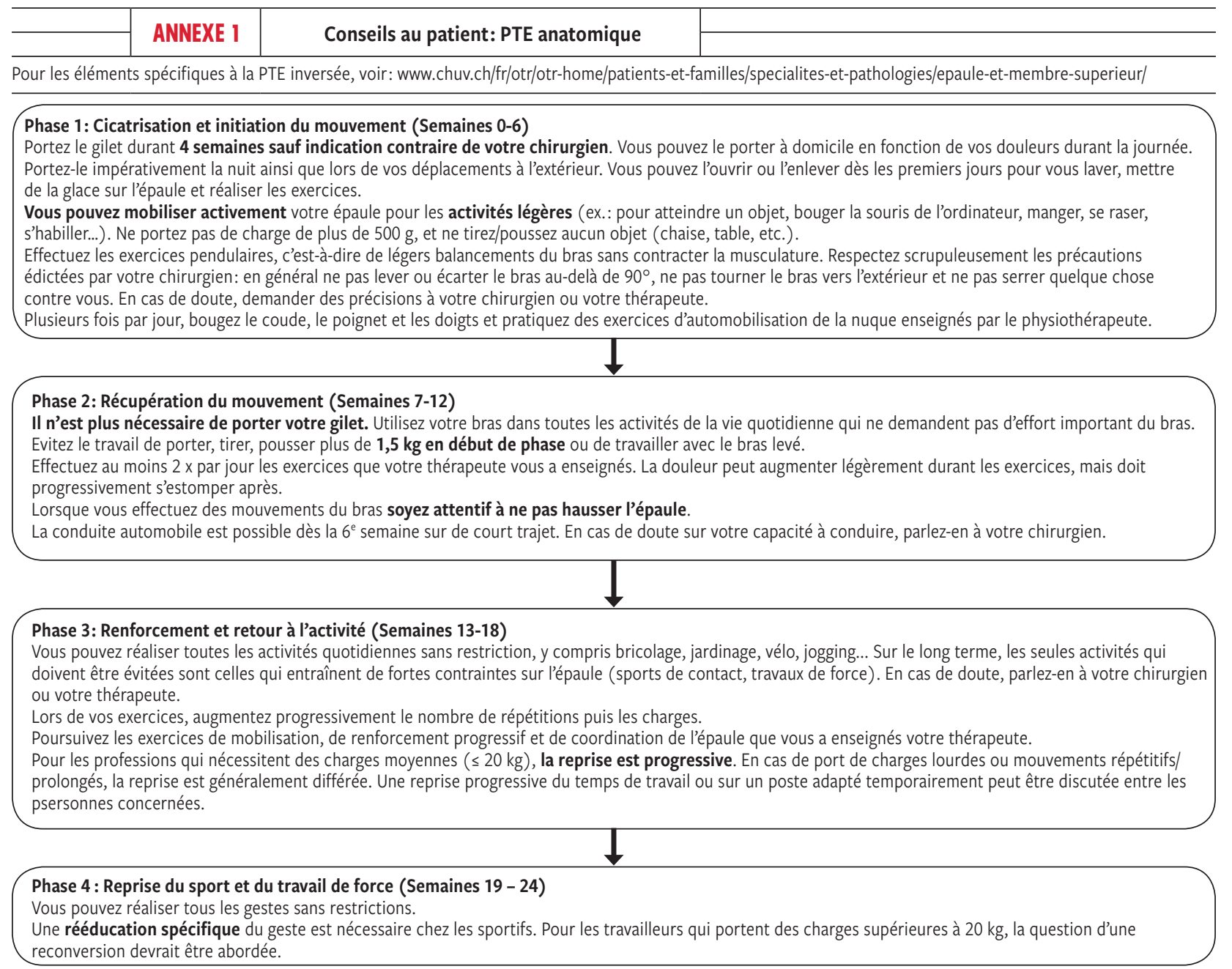

